\title{
Synthesis and Characterization of Fused Pyrimido-pyrimidine Dicarbonitriles and their Antibacterial Activity
}

\author{
G. S.WAGHMARE, S. B. JUNNE, S. D. SHINDE, \\ A. S. WAGHAMARE and S.V. KUBERKAR*
}

Organic Synthesis Laboratory, Post Graduate Department of Chemistry, Yeshwant Mahavidyalaya, Nanded-05 (M.S.), India

gswagh@yahoo.co.in

Received 6 May 2012 / Accepted 28 May 2012

\begin{abstract}
Novel heterocyclic compounds containing pyrimido-pyrimidine moiety have been synthesized by the reaction of 2,4-diamino-6-(4'-substituted phenyl)pyrimidine-5-carbonitrile with bis-(-methylthio)methylene malanonitrile in presence of anhydrous $\mathrm{K}_{2} \mathrm{CO}_{3}$ to yield 6-amino-4-imino-8-(-4'-substituted phenyl)-2-(methylthio) 4(H) pyrimido[1,2-b]pyrimidine-3,7dicarbonitrle. The structure of pyrimido-pyrimidine dicarbonitriles have been characterized by using IR and ${ }^{1} \mathrm{H}$ NMR spectroscopy. These compounds were screened for their antibacterial activity.
\end{abstract}

Keywords: 2,4-Diamino-6-(4'-substituted phenyl)pyrimidine-5-carbonitrile, Bis-(methylthio)methylene malanonitrile, Anhydrous $\mathrm{K}_{2} \mathrm{CO}_{3}$, Antibacterial activity, Penicillin

\section{Introduction}

In the last few years researchers have been highly interested in the chemistry of heterocyclic derivatives with their expected biological activity ${ }^{1-3}$. Earlier of this fused pyrimido benzothiazole possessing three to four rings ${ }^{4-6}$ have been reported, which exhibit the activities like Anti-inflammatory ${ }^{7}$, antiallergic ${ }^{8}$, antitumer ${ }^{9}$ and antiparakinsonism ${ }^{10}$ some pyrimidine derivatives showed antihypertensive, antipyretics, analgesics ${ }^{11}$ activity.

Few pyrimidine derivatives are pesticides ${ }^{12}$, herbicides and plant growth regulators ${ }^{13}$. Synthetic methodologies for the synthesis of novel fused pyrimido-pyrimidine nuclous have better interest in pharmaceutical and biological activity, particularly in cancer research.

In this route, we report the novel heterocyclic system possessing fused two rings. 6-Amino-4-imino-8-(4'substituted phenyl)-2-(methylthio)-4(H) pyrimido[1,2-b]pyrimidine3,7-dicarbonitrile (III) was synthesized from 2,4 diamino-6-(-4'-substituted phenyl) pyrimidine-5-carbonitrile (I) ${ }^{14}$ with bis-(methyl thio)methylene malanonitrile (II) in DMF solvent in presence of anhydrous $\mathrm{K}_{2} \mathrm{CO}_{3}$. 
<smiles>[R]c1ccc(-c2nc(N)nc(N)c2C#N)cc1</smiles>

( I)

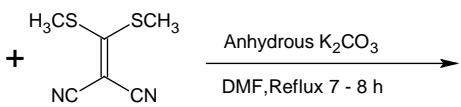

( II)

\section{Scheme 1}<smiles>[R]c1ccc(-c2nc3nc(S)c(C#N)c(=N)n3c(N)c2C#N)cc1</smiles>

(III a - e )

\section{Experimental}

Melting points were determined on a Gallenkamp melting point apparatus and are uncorrected (Table 1). All products were monitored by TLC using Merck silica gel for TLC. IR spectra were measured on SHIMADZU FTIR spectrometer. ${ }^{1} \mathrm{H}$ NMR spectra were recorded on a BRUKER DRX $300 \mathrm{~A} . \mathrm{V}$. ANCE spectrometer at $300 \mathrm{MHz}$. Mass spectra was recorded on a SHIMADZU QP1100EX mass spectrometer.

Preparation of 6-amino-4-imino-8-(4'-substituted phenyl)-2-(methylthio)-4-(H) pyrimido[1,2-b]pyrimidine-3,7 dicarbonitrile (III a-e $_{\text {) }}$

Mixtures of compound (I) (2.4 g 1 eq) and reagent compound (II) (1.4 g 1 eq) in $25 \mathrm{~mL}$ DMF was refluxed for 6-8 $\mathrm{h}$ in presence of anhydrous $\mathrm{K}_{2} \mathrm{CO}_{3}$. The progress of the reaction was monitored by TLC using benzene:ethyl acetate. The reaction mixture was transferred into ice cold water, solid gets separated out and recrystallized from ethanol (Scheme 1). The yield is shown in Table 2.

Table 1. Physical data and elemental analysis

\begin{tabular}{|c|c|c|c|c|c|c|c|}
\hline \multirow{2}{*}{$\mathrm{R}$} & \multirow{2}{*}{ Compd. } & \multirow{2}{*}{$\begin{array}{c}\text { Mol. } \\
\text { formula }\end{array}$} & \multicolumn{2}{|c|}{ Mol. M.P. } & \multirow{2}{*}{$\begin{array}{l}\text { Yield } \\
\%\end{array}$} & \multicolumn{2}{|c|}{ Elemental analysis \% } \\
\hline & & & $\mathrm{Wt}$ & ${ }^{\circ} \mathrm{C}$ & & Calculated & Found \\
\hline \multirow{3}{*}{$\mathrm{H}$} & \multirow{3}{*}{ III $_{\mathbf{a}}$} & \multirow{3}{*}{$\mathrm{C}_{16} \mathrm{H}_{11} \mathrm{~N}_{7} \mathrm{~S}$} & \multirow{3}{*}{333} & \multirow{3}{*}{260} & \multirow{3}{*}{60} & $\mathrm{C}=57.65, \mathrm{H}=3.30$ & $\mathrm{C}=57.70, \mathrm{H}=3.31$ \\
\hline & & & & & & $\mathrm{N}=29.42, \mathrm{~S}=9.60$ & $\mathrm{~N}=29.40, \mathrm{~S}=9.61$ \\
\hline & & & & & & $C=56.19, S=8.81$ & $C=56.20, S=8.85$ \\
\hline $\mathrm{OMe}$ & III $_{b}$ & $\mathrm{C}_{17} \mathrm{H}_{13} \mathrm{~N}_{7} \mathrm{SO}$ & 363 & 240 & 85 & $\begin{array}{l}\mathrm{N}=26.99, \mathrm{H}=3.58, \\
\mathrm{O}=4.40\end{array}$ & $\begin{array}{l}\mathrm{N}=26.99, \mathrm{H}=3.59 \\
\mathrm{O}=4.40\end{array}$ \\
\hline $\mathrm{CH}_{3}$ & III $_{c}$ & $\mathrm{C}_{17} \mathrm{H}_{13} \mathrm{NS}$ & 347 & 210 & 72 & $\begin{array}{l}C=58.78, N=28.24, \\
H=3.74, S=9.22\end{array}$ & $\begin{array}{l}C=58.78, N=28.24, \\
H=3.75, S=9.25\end{array}$ \\
\hline $\mathrm{Cl}$ & III $_{d}$ & $\mathrm{C}_{16} \mathrm{H}_{10} \mathrm{~N}_{7} \mathrm{SCl}$ & 367 & 250 & 50 & $\begin{array}{l}\mathrm{C}=52.31, \mathrm{~N}=26.70, \\
\mathrm{H}=2.72, \mathrm{~S}=8.71, \\
\mathrm{Cl}=9.67\end{array}$ & $\begin{array}{l}\mathrm{C}=52.30, \mathrm{~N}=26.71, \\
\mathrm{H}=2.73, \mathrm{~S}=8.72, \\
\mathrm{Cl}=9.68\end{array}$ \\
\hline $\mathrm{NO}_{2}$ & III $_{e}$ & $\mathrm{C}_{16} \mathrm{H}_{10} \mathrm{~N}_{7} \mathrm{SO}_{2}$ & 364 & 260 & 62 & $\begin{array}{l}\mathrm{C}=52.74, \mathrm{~N}=26.92, \\
\mathrm{O}=8.79, \mathrm{~S}=8.79, \\
\mathrm{H}=2.74\end{array}$ & $\begin{array}{l}\mathrm{C}=52.75, \mathrm{~N}=26.92, \\
\mathrm{O}=8.80, \mathrm{~S}=8.80, \\
\mathrm{H}=2.74\end{array}$ \\
\hline
\end{tabular}

Table 2. Reaction time and yield

\begin{tabular}{cccc}
\hline Compounds & $\mathrm{R}$ & Reflux Time, $\mathrm{h}$ & Yield, \% \\
\hline $\mathbf{I I I}_{\mathbf{a}}$ & $\mathrm{H}$ & 7 & 60 \\
$\mathbf{I I I}_{\mathbf{b}}$ & $\mathrm{Ome}$ & 6 & 85 \\
$\mathbf{I I I}_{\mathbf{c}}$ & $\mathrm{CH}$ & $6-7$ & 72 \\
$\mathbf{I I I}_{\mathbf{d}}$ & $\mathrm{Cl}$ & 8 & 50 \\
$\mathbf{I I I}_{\mathbf{e}}$ & $\mathrm{NO}_{2}$ & 8 & 62 \\
\hline
\end{tabular}




\section{Spectral analysis}

6-Amino-4-imino-8-phyenyl-2-(methylthio)-4(H)pyrimido[1,2-b]pyrimidine3-7, dicarbonitrile $\left(\mathbf{I I I}_{a}\right)$

Red brown crystals; mp $=260{ }^{\circ} \mathrm{C}$, IR: $v_{\max }(\mathrm{KBr})$ 3380, $3329\left(\mathrm{NH}_{2}\right), 2210(\mathrm{CN}), 1680$ $(\mathrm{C}=\mathrm{N}), 2980,2850\left(-\mathrm{CH}_{3}\right)$ 1620, 1580,1540 (Ar) $\mathrm{cm}^{-1}$; ${ }^{1} \mathrm{H}$ NMR: (300 MHz, DMSO-d6) $\delta$ $2.5 \mathrm{~s}(3 \mathrm{H}), 4.2 \mathrm{~s}\left(\mathrm{NH}_{2}\right), 6.8-7.5 \mathrm{~m}(\mathrm{Ar}-\mathrm{H})$. MS, m/z, $330\left(\mathrm{M}-2, \mathrm{NH}_{2}\right) \&(\mathrm{M}-1, \mathrm{NH})$.

6-Amino-4-imino-8-(4'-methoxy phenyl)-2-(methylthio)-4(H) pyrimido[1,2-b]pyrimidine $3-7$, dicarbonitrile ( III $_{\mathbf{b}}$ )

Red brown colour; mp $=240{ }^{\circ} \mathrm{C}$, IR: $v_{\max }(\mathrm{KBr}) 3401,3350\left(\mathrm{NH}_{2}\right), 2202(-\mathrm{CN}), 1630(\mathrm{C}=\mathrm{N})$, 2995,2890 $\left(-\mathrm{CH}_{3}\right)$ 1590, 1550,1420 (Ar-H) $\mathrm{cm}^{-1} ;{ }^{1} \mathrm{H}$ NMR: $\delta 2.19 \mathrm{~s}(3 \mathrm{H}), 3.63 \mathrm{~s}(3 \mathrm{H}), 4.5 \mathrm{~s}$ $\left(\mathrm{NH}_{2}\right)$, 6.5-7.9 m (Ar-H). MS, $m / z, 330\left(m-\mathrm{OCH}_{3} \& \mathrm{M}^{-\mathrm{NH}_{2}}\right)$.

6-Amino-4-imino-8-(4'-methyl phenyl)-2-(methylthio)-4(H) pyrimido[1,2-b]pyrimidine 3,7, dicarbonitrile (III $)$

$\mathrm{Mp}=210{ }^{\circ} \mathrm{C}$, IR: $v_{\max }(\mathrm{KBr})$ 3412, $3380\left(-\mathrm{NH}_{2}\right), 2950,2890\left(\mathrm{CH}_{3}\right), 2215(\mathrm{CN}), 1625$ $(\mathrm{C}=\mathrm{N}), 1620,1580,1400(\mathrm{Ar}-\mathrm{H}) ;{ }^{1} \mathrm{H}$ NMR: $\delta 2.25 \mathrm{~s}(3 \mathrm{H}), 2.95 \mathrm{~s}(3 \mathrm{H}), 4.31 \mathrm{~s}\left(\mathrm{NH}_{2}\right), 6.8-$ $7.9 \mathrm{~m}$ (Ar-H).

6-Amino-4-imino-8-(4'-chlorophenyl)-2-(methylthio)-4(H) pyrimido[1,2-b]pyrimidine 3,7, dicarbonitrile $\left(\mathbf{I I I}_{\mathbf{d}}\right)$

$\mathrm{Mp}=250{ }^{\circ} \mathrm{C}$, IR: $v_{\max }(\mathrm{KBr})$ 3401, $3350\left(-\mathrm{NH}_{2}\right), 2960,2850\left(\mathrm{CH}_{3}\right), 2220(\mathrm{CN}), 1630(\mathrm{C}=\mathrm{N})$, 1615,1590,1510 (Ar-H); ${ }^{1} \mathrm{H}$ NMR: $\delta 2.30 \mathrm{~s}(3 \mathrm{H}), 4.1 \mathrm{~s}\left(\mathrm{NH}_{2}\right)$, 6.5-7.6 m (Ar-H).

6-Amino-4-imino-8-(4'-Nitrophenyl) 2-(methylthio) 4(H) pyrimido[1,2-b]pyrimidine 3,7, dicusbonitrile $\left(\mathbf{I I I}_{\boldsymbol{e}}\right)$

Yellow crystal, mp $=260{ }^{\circ} \mathrm{C}$, IR: $v_{\max }(\mathrm{KBr}) 3409,3380\left(-\mathrm{NH}_{2}\right), 2981,2850\left(-\mathrm{CH}_{3}\right), 2210$ (CN), $1640(\mathrm{C}=\mathrm{N}), 1625,1595,1520(\mathrm{Ar}-\mathrm{H}), 1518,1420\left(-\mathrm{NO}_{2}\right){ }^{1} \mathrm{H}$ NMR: $\delta 2.10 \mathrm{~s}(3 \mathrm{H}), 4.5$ $\left(\mathrm{NH}_{2}\right)$, 6.6-7.8 m (Ar-H).

\section{Results and Discussion}

Reaction of bis(methylthio)methylene malanonitrile (II) (1 eq.) with 2.4 diamino-6-(4'substituted phenyl)pyrimidine-5-carbonitrile (I) (1.eq) in presence of anhydrous $\mathrm{K}_{2} \mathrm{CO}_{3}$ and DMF as solvent was carried out under reflux for 7-8 hours to yield compound $\mathbf{I I I}_{\mathbf{a}-\mathrm{e}}$.

The compound (I) having 2,4-diamino functional group but amino group at $2^{\text {nd }}$ position become free from steric hindrance, under maintained condition and equivalent ratio monocyclisation occurs to form compound $\mathbf{I I I}_{\mathbf{a} \text {-e. }}$. After spectral studies monocyclised compounds were found and the yield of compounds is shown in the Table 2.

\section{Antibacterial activity}

The synthesized compounds were screened for their antibacterial activity against gram positive species S.aureus and B.substilis and gram-negative species E.coli and S.typhi by paper disc diffusion method ${ }^{15}$. All the synthesized compounds were dissolved in dimethyl formamide (DMF). The synthesized compounds showed zone of inhibition from 8-14 mm in diameter, whereas standard streptomycin exhibit zone of inhibition is 16 and $20 \mathrm{~mm}$ in diameter against $S$. aureus and B.substilis and penicillin exhibited zone of inhibition is 13 \& 
$16 \mathrm{~mm}$ in diameter against E.coli and S.typhi respectively. Among all synthesized compounds III $_{\mathbf{a}-\mathbf{e}}$, compound III $_{\mathbf{d}}(12,10,12,13)$ and compound III $_{\mathbf{b}}(\mathbf{0 9}, 11,11,14)$ showed higher zone of inhibition against S.aureus, B.substilis, E.coli and S.typhi respectively (Table 3). Our conclusion about present work showed that presence of halogen $(\mathrm{Cl})$ and methoxy $\left(\mathrm{OCH}_{3}\right)$ group at 4'-position increases the antibacterial activity.

Table 3. Antibacterial activity of compounds ( III $\left._{\mathbf{a}-\mathrm{e}}\right)$

\begin{tabular}{ccccc}
\hline \multirow{2}{*}{ Compd. } & \multicolumn{4}{c}{ Zone of inhibitation in dimeter in mm at $25 \mu \mathrm{g} /$ disc } \\
\cline { 2 - 5 } & S.aureus & B.substilis & E.coli & S.typhi \\
\hline III $_{\mathbf{a}}$ & 09 & 10 & 08 & 09 \\
III $_{\mathbf{b}}$ & 09 & 11 & 11 & 14 \\
III $_{\mathbf{c}}$ & 08 & 10 & 09 & 12 \\
III $_{\mathbf{d}}$ & 12 & 10 & 12 & 13 \\
III $_{\mathbf{e}}$ & 11 & 09 & 08 & 10 \\
Streptomycin & 16 & 20 & - & - \\
Penicillin & -- & - & 13 & 16 \\
\hline
\end{tabular}

\section{Acknowledgement}

The authors are thankful to the Principal, Yeshwant Mahavidyala, Nanded for providing laboratory and IR facilities for research work. Authors are also thankful to the Director IICT Hyderabad for providing spectral analysis.

\section{References}

1. Attaby F A, Eldin S M and Razik M A, Phosphorus Sulfur, 1995, 106, 21.

2. Ghabrial S S and Eldin S M, Egypt J Pharm Sci., 1996, 37, 375.

3. Ghabrial S S, Zaki M Y and Eldin S M, Indian J Chem., 1994, 33B, 855.

4. Baheti K G, Jadhav J S, Suryavanshi A T and Kuberkar S V, Indian J Chem., 2005, 44B, 834-837.

5. Gompper R and Topfi W, Chem Ber., 1962, 95, 2871.

6. $\quad$ Alaimo R J, J Heterocycl Chem., 1973, 10(5), 769-772.

7. Glennon R A, Gaines J J and Rogers M E, J Med Chem., 1981, 24(6), 766.

8. Victor S, Eur Pat., 1981, 21807, Chem Abstr., 1981, 94, 208900s.

9. $\quad$ Peter H L, U S Pat, 1972, 3704303, Chem Abstr., 1972, 78, 43513x.

10. Wade J J, Toso C B, Mastson C J and Stelzer V L, J Med Chem., 1983, 26, 608.

11. Hassan N A, Molecule, 2000, 5, 827.

12. Nega S, Aionso J, Diazj A and Junquere F, J Heterocycl Chem., 1990, 27, 269.

13. Shishoo C J and Jain K S, J Heterocycl Chem., 1992, 29, 883-893.

14. Hassan Sheibani, Arman S Saljoogi and Ayoob Bazgir, ARKIVOC, 2008, (II), 115-123.

15. Ananthanarayan R and Panikar J C K, Text Book of Microbiology, Orient Longman, 1999, 578. 